\title{
Mathematical Model to Estimate and Predict the COVID-19 Infections in Morocco: Optimal Control Strategy
}

\author{
Omar Zakary $D^{D}$, Sara Bidah $(D$, , Mostafa Rachik $(\mathbb{D}$, and Hanane Ferjouchia \\ Laboratory of Analysis Modelling and Simulation, Department of Mathematics and Computer Science, Faculty of Sciences Ben M'Sik, \\ Hassan II University of Casablanca, BP 7955, Sidi Othman, Casablanca, Morocco \\ Correspondence should be addressed to Omar Zakary; zakaryma@gmail.com
}

Received 10 April 2020; Revised 18 June 2020; Accepted 5 August 2020; Published 9 October 2020

Academic Editor: Sazzad Hossien Chowdhury

Copyright (C) 2020 Omar Zakary et al. This is an open access article distributed under the Creative Commons Attribution License, which permits unrestricted use, distribution, and reproduction in any medium, provided the original work is properly cited.

\begin{abstract}
In this paper, we aim to estimate and predict the situation of the new coronavirus pandemic (COVID-19) in countries under quarantine measures. First, we present a new discrete-time mathematical model describing the evolution of the COVID-19 in a population under quarantine. We are motivated by the growing numbers of infections and deaths in countries under quarantine to investigate potential causes. We consider two new classes of people, those who respect the quarantine and stay at home, and those who do not respect the quarantine and leave their homes for one or another reason. Second, we use real published data to estimate the parameters of the model, and then, we estimate these populations in Morocco. We investigate the impact of people who underestimate the quarantine by considering an optimal control strategy to reduce this category and then reducing the number of the population at risk in Morocco. We provide several simulations to support our findings.
\end{abstract}

\section{Introduction}

Coronaviruses $(\mathrm{CoV})$ are a large family of zoonotic viruses, that is, they are transmitted from animals to humans, and that cause symptoms ranging from the common cold to more serious illnesses such as Middle East Respiratory Syndrome (MERS) which is transmitted from dromedary to humans and severe acute respiratory syndrome (SARS) which is transmitted from civet to humans. Several known coronaviruses that have not yet infected humans are circulating in some animals [1].

The new coronavirus SARS-CoV-2 is a new strain of coronaviruses that have not yet been identified in humans, where respiratory symptoms, fever, cough, shortness of breath, and dyspnea, are common signs of the infection with the new coronavirus pandemic, COVID-19. In more severe cases, this disease can cause pneumonia, severe acute respiratory syndrome, kidney failure, and even death [1].

The WHO standard recommendations to the general public for reducing exposure to, and transmission of, a range of diseases are the following and include hand hygiene, respiratory hygiene, and good food safety practices [1]: (i) Wash your hands frequently with a hydroalcoholic solution or with soap and water

(ii) Cover your mouth and nose with the crease of your elbow or with a handkerchief if you cough or sneeze; throw the handkerchief immediately after and wash your hands

(iii) Avoid close contact with people who have a fever and cough

(iv) In case of fever, cough, and difficulty in breathing, consult a doctor without delay and tell him the trips made

(v) In markets located in areas where there are currently cases of the new coronavirus, avoid unprotected direct contact with live animals and with surfaces in contact with animals

(vi) Consumption of raw or undercooked animal products should be avoided. In accordance with good food safety practice, raw meat, milk, or organ meats should be handled with care to avoid crosscontamination with raw food 
At the moment, there is no vaccine for the new coronavirus SARS-CoV-2, because in general, when a new disease appears, a vaccine only becomes available after a vaccine development process, which can take several years. Furthermore, there is no specific treatment for the disease resulting from the new coronavirus. However, many symptoms can be treated, and treatment therefore depends on the clinical condition of the patients. In addition, providing supportive care to infected people can be very effective [2].

Countries all over the world are intensifying their efforts to combat the COVID-19, which first appeared in the Chinese city of Wuhan, and that has killed at least 100,000 people. More than 1.5 million cases have been reported worldwide, most of them in the United States, Italy, Spain, and China. A man from Wuhan died in the Philippines on February 2, and Hong Kong reported the first death on February 4. The infection is now more widespread than the 2002-2003 severe acute respiratory syndrome (SARS) epidemic, also of Chinese origin, in terms of people affected and deaths.

Outbreaks of novel virus infections among people are always of public health concern, especially when there is little knowledge about the characteristics of the virus, how it spreads between people, how severe are the resulting infections, and how to treat them.

The mathematical modeling is one of the most solutions that can respond to all these questions. In the absence of vaccines or antivirals for the COVID-19, the effective implementation of nondrug interventions, such as personal protection and social exclusion, will be crucial in controlling the epidemic [3-5].

From the point of view of additional resources to fight the epidemic, mathematics and statistical modeling can be useful in creating forecasts for the cases announced. These expectations may include estimates of the burden of illness which can help guide public health officials in preparing for medical care and other resources needed to combat the epidemic. Predictions can also guide the severity and the type of interventions needed to mitigate the epidemic.

In this contribution, we develop a mathematical model that describes the evolution of the COVID-19 in countries under quarantine. Here, we use actual data from March 19 to April 05, 2020, of daily updates laboratory-confirmed cases, recovered, and deaths in Morocco from the Johns Hopkins University Center for Systems Science and Engineering (JHU CCSE) [6], in order to estimate the parameters of the model and then predict the severity of the possible infection in the coming months. Using this method, we can estimate the total population at risk in Morocco and justify the growing number of new confirmed cases despite the nationwide quarantine. Finally, we investigate an optimal control strategy that aims to reduce the population at risk in Morocco, whereas the control strategy we are proposing here adopts nondrug intervention which makes it very optimal and more practical to use in developing countries.

The paper is organized as follows: Section 2 introduces our new model, giving some details about interactions between the different compartments and parameters of the model. In Section 3, we provide the results of the parameters' estimation validating our model and predicting the total population at risk in Morocco. In Section 4, we present the optimal control problem to justify the existence and the characterization of the optimal control. Numerical simulations are carried out in Section 5. In Section 6, we provide a global sensitivity analysis, and Section 7 concludes.

\section{Methods}

2.1. Background. In late December 2019, several local health facilities reported groups of patients with pneumonia for unknown reasons linked to the seafood and wholesale wet animal market in Wuhan, Hubei Province, China. On December 31, 2019, the Chinese Center for Disease Control and Prevention (CDC) dispatched a rapid response team to support health authorities in Hubei Province and Wuhan City and conduct an epidemiological investigation to locate the source of pneumonia groups. This led to a description of a new coronavirus found in samples of pneumonia patients at the beginning of the epidemic [7]. Less than a month after identifying patients, on January 1,2020 , the government closed this seafood market [8]. A consortium led by a Chinese scientist and international researchers quickly gathered to intensify national and international efforts and coordination. On January 10, 2020, it released a partial sequence obtained from a patient from Wuhan who presented at least $70 \%$ similarity in genetic material to severe acute respiratory syndrome (SARS) [8].

Based on previous experiences with respiratory infections, the Chinese hospitals recognized the utmost importance of infection control preparedness in their healthcare system. Standby level includes alert, dangerous level 1, dangerous level 2, and emergencies; the activation level is determined according to the risk assessment. Infection control measures and administrative support are strengthened with an indication of different levels of preparedness [9]. Through this infrastructure, China has overcome the challenge of influenza A pandemic in $2009[10,11]$ and the emergence of bird flu A H7N9 in 2013 [12].

To prepare for this emerging infectious disease, fever screening was done at the airports and at the high-speed train stations, with special emphasis on flights and trains from Wuhan, while passengers with a fever above $38^{\circ} \mathrm{C}$ are referred to public hospitals for assessment. In addition, several control measures are implemented in the public hospital system [9].

Many countries have taken advantage of the experience of China in fighting this epidemic. This saved them time to try to contain the spread of the COVID-19 infection and learn them that this spare time must be used efficiently and effectively. Morocco is one of these countries, whereas Morocco was proactive in declaring a health emergency on March 20, at 6:00 pm until further notice, in response to the growing threat of a new coronary virus (COVID-19), after the Ministry of Health confirmed case 63 of COVID-19, two patients recovered and two died. The state of emergency means that citizens cannot go out to public places without special permission from the local authorities. The state of emergency allows the government to take tougher measures to respond to the epidemic and to release budgets to improve 
medical care to impose closures on whole cities. When all international flights to and from Moroccan territory are suspended until further notice, all public gatherings of more than 50 people are prohibited, including economic and political meetings, cultural and religious ceremonies, sporting events, and other gathering places closed. Courses have been canceled in all institutions - from daycare centers to universities, from public to private- until further notice $[13,14]$.

2.2. The Model. Despite all of the efforts made by the authorities, the number of confirmed COVID-19 cases is still rising, which means that something is wrong. One possible reason is the lack of respect for the national quarantine and the underestimation of the contagiousness of the SARS-CoV-2.

In the literature, over the past three months, several works of mathematical modeling have been published taking into account the exposed and asymptomatic classes [15-19]. This motivated us to consider more general classes in order to study the efficiency of the national closure and to evaluate our strategy of control that will be applied to all people regardless if they are exposed or not.

The first class is the aware individuals who know about the virus and the nationwide lockdown, called the partially controlled individuals $(\mathrm{P})$, because this population could not respect the national quarantine for one reason or another and could be considered as law violators.

The second category is people who respect the national quarantine by staying at home from the start of the closure. This population is called a totally controlled population (C). Susceptible, infected, recovered, and dead individuals are the rest of the classes studied here.

Let us define a discrete-time model with the six following main compartments:

(i) S: the number of susceptible people to infection or who are not yet infected, and people that did not benefit from the awareness program

(ii) P: the number of susceptible people who are partially controlled. People who create arguments to leave their homes during the quarantine and can, therefore, be considered as people not respecting the quarantine

(iii) C: the number of susceptible people who are totally controlled. Those who do not leave their home during quarantine and can, therefore, be considered as people respect quarantine

(iv) I: the number of infected people who are capable of spreading the epidemic to those in the susceptible and partially controlled categories

(v) R: the number of recovered people from the epidemic, but can return to the susceptible class because of the short-term removal of individuals' immunity

(vi) D: the death toll from COVID-19

In discrete-time models, we can choose the unit of time we need, a day, a week, or a month, and we collect all the events from time step $i$ to time step $i+1$ as events at the same time. In our model, we choose the unit of time as a day, since COVID-19 data is collected daily in almost all countries of the world. All these considerations lead to the following system of difference equations:

$$
\begin{aligned}
& S_{i+1}=S_{i}-\frac{\beta}{N_{i}} S_{i} I_{i}-\theta \frac{S_{i}}{1+a S_{i}}+\rho R_{i}-u S_{i}, \\
& P_{i+1}=P_{i}-\frac{c \beta}{N_{i}} P_{i} I_{i}+\theta_{1} \frac{S_{i}}{1+a S_{i}}-u P_{i}, \\
& C_{i+1}=C_{i}+\theta_{2} \frac{S_{i}}{1+a S_{i}}+u P_{i}+u S_{i}, \\
& I_{i+1}=I_{i}+\frac{\beta}{N_{i}}\left(S_{i}+c P_{i}\right) I_{i}-\gamma I_{i}-\alpha I_{i}-\mathscr{T} I_{i}, \\
& R_{i+1}=R_{i}+\mathscr{T} I_{i}+\gamma I_{i}-\rho R_{i}, \\
& D_{i+1}=D_{i}+\alpha I_{i},
\end{aligned}
$$

with initial conditions $S_{0} \geq 0, P_{0} \geq 0, C_{0} \geq 0, I_{0} \geq 0, R_{0} \geq 0$, and $D_{0} \geq 0$ and where $N_{i}=S_{i}+P_{i}+C_{i}+I_{i}+R_{i}$ is the total size of the population at the instant $i$. Without loss of generality, we neglect the recruitment rate and the natural death rate due to the restricted time window of the study. $\theta=\theta_{1}$ $+\theta_{2}$ defines the awareness control parameter, where $\theta_{1}$ is the recruitment rate of susceptibles to the partially controlled class and $\theta_{2}$ is the recruitment rate of susceptibles to the totally controlled class. $a$ is the inhibition effect due to resource limitation to awareness controls, $\beta$ is the infection transmission rate, with " $c$ " modeling the reduced chances of a partially controlled individual to be infected where $0 \leq c$ $\leq 1, \mathscr{T}$ is the treatment rate, $\alpha$ is the infection death rate, $\gamma$ is the recovery rate, and $\rho$ is the losing removal individuals' immunity rate.

We note that the population size $N_{i}$ is not constant in time $i$, in fact

$$
\begin{aligned}
& N_{i+1}=S_{i+1}+P_{i+1}+C_{i+1}+I_{i+1}+R_{i+1}, \\
& N_{i+1}=N_{i}+\Gamma_{i}-\mu N_{i}-\alpha I_{i}, \\
& N_{i+1}=N_{i}-\alpha I_{i} .
\end{aligned}
$$

Hence, the population decreases until the end of the epidemic. A summary of the description and values of the parameters is given in Table 1. To the authors' knowledge, the model we propose in this paper is the first discrete-time mathematical model which considers people who respect and people who do not respect the state of health emergency as new compartments.

\section{Estimation of the Parameters}

Johns Hopkins University Center for Systems Science and Engineering (JHU CCSE) provides available data for academic research purposes from multiple sources [6]. Data information includes cumulative confirmed cases, the cumulative number of cases recovered, and the cumulative number 
TABle 1: Parameter descriptions and values; the initial data are corresponding to the 19 March 2020 for Morocco [20].

\begin{tabular}{lcc}
\hline Parameter & Description & Estimated values for Morocco \\
\hline$\beta$ & The infection rate & 0.309038973249694 \\
$c$ & The reduced chances of a partially controlled individual to be infected & 0.790453244925308 \\
$\theta_{1}$ & The recruitment rate of susceptibles to the partially controlled class & 0.198352095739391 \\
$\theta_{2}$ & The recruitment rate of susceptibles to the totally controlled class & 0.0609987020406361 \\
$\alpha$ & Death due to the infection & 0.010653085736073 \\
$\mathscr{T}$ & Treatment rate & 0.00853317853991 \\
$\gamma$ & Recovery rate & 0.00112696312179574 \\
$\rho$ & Ime inhibition effect due to resource limitation to awareness controls & $3.07478906124174 \times 10^{-14}$ \\
$a$ & The effect of the lockdown & $8.77262435783777 \times 10^{-8}$ \\
$u$ & Initial susceptible population & 0.0372352811096721 \\
$S_{0}$ & Initial partially controlled population & 34500000 \\
$P_{0}$ & Initial totally controlled population & 0 \\
$C_{0}$ & Initial infected population & 0 \\
$I_{0}$ & Initial removed population & 63 \\
$R_{0}$ & Initial dead population & 2 \\
$D_{0}$ & & 2
\end{tabular}

of deaths. To get an insight into the evolution of the COVID19 in Morocco, we have acquired updated data on the cumulative number of laboratory-confirmed cases for COVID-19 from the CSSE data of [6] to estimate the parameters of the model (1), (2), (3), (4), (5) and (6). For more details about the available data, see [6].

The following process has been followed for parameters estimation: using MATLAB, the system of difference equations is solved numerically, with initial chosen values for parameters and state variables from Table 1. Model outcomes are compared with the field data, and the LevenbergMarquardt optimization algorithm determines a new set of parameters' values with the model outcomes in a better fit to the field data $[21,22]$. After new parameters' values are determined by this optimizer, the system of difference equations is solved numerically using these new parameters' value, and the model outcomes are compared again with the field data. This iteration process between parameter updating and numerical solutions of the system of difference equations continue till convergence criteria for the parameters are met. In this process of estimating, parameters about one thousand values are chosen using a random process for each of the parameters to be estimated.

From Figure 1, we can see that the models (1), (2), (3), (4), (5) and (6) correctly correspond to the actual data especially the infections curve, where we can see the increase of active cases in Morocco, despite the nationwide quarantine. We can also see that the number of individuals who have recovered and died continues to rise slightly compared to the number of infections that amounted to about 1,000 on April 05. Based on the estimated parameters, we simulate the models (1), (2), (3), (4), (5) and (6) from March 19 to May 07 to predict the course of the events and to see if the status of the COVID-19 infections in Morocco will reach the peak or not. It can be seen from Figure 2 that by May 07, the number of active cases in Morocco will exceed 3000 individuals, more than 600 recovered individuals, and about 800 deaths. This simulation shows the efficiency of the strategy of control used by the Moroccan authorities, by slowing the spread of the epidemic compared to other affected countries such as Italy, Spain, and the United States that are in the top of the list of affected countries. To justify the increase of the number of infections in Morocco despite the use of the national quarantine, we estimate the number of people who do not respect and those respecting the quarantine in Figure 3, where we can see that the number of totally controlled individuals starts increasing slowly from the beginning of the closure on March 20 to reach about 30 million at the beginning of May. But the number of partially controlled individuals rises continuously to reach about 15 million individuals before starting decreasing towards 5 million by the first week of May. That means that the population at risk in Morocco in the first month of the national closure is more than ten million. This result explains the growing number of infections and deaths in the country despite the nationwide quarantine.

We determine numerically the residuals of the parameters' estimation, and then, we examine the accuracy of the normality of the estimation of the parameters. In order to carry out this examination, we generate the residuals of the three functions of infected, removed, and dead populations, depicted in Figure 4, where it can be seen that there are small residuals compared to the values of each population. While Figure 5 displays the corresponding histograms of $I_{k}-I_{k}^{\text {data }}$ in (a) and $R_{k}-R_{k}^{\text {data }}$ in (b) and $D_{k}-D_{k}^{\text {data }}$ in (c) for the estimation of the parameters for Morocco with their corresponding normal distributions. These figures show that the estimated parameters are reliable and correspond correctly 


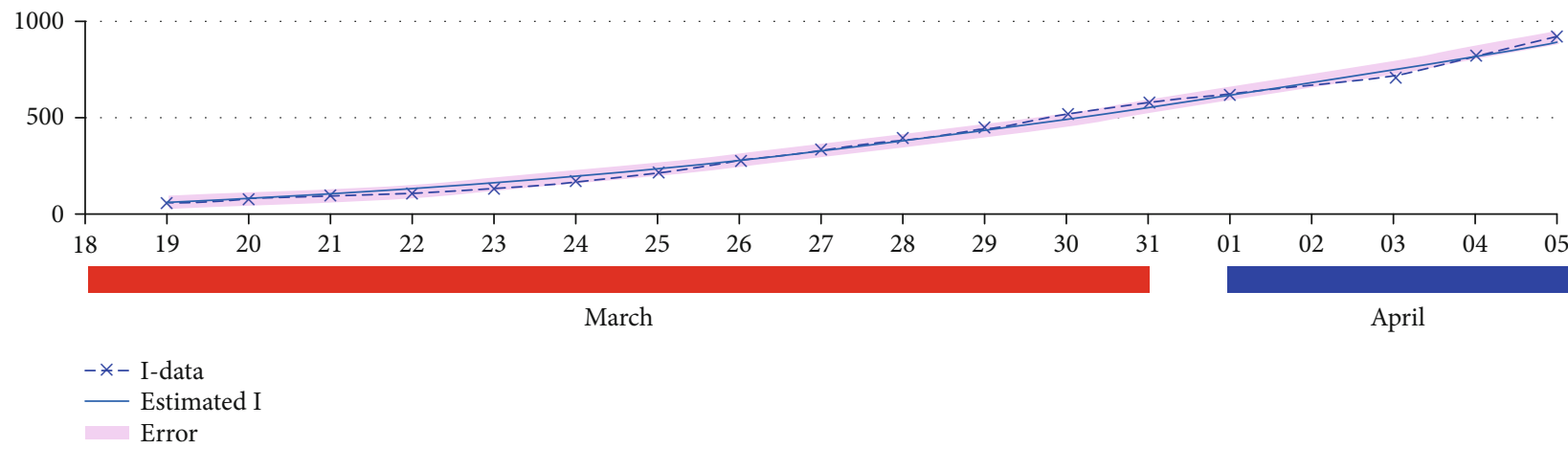

(a)

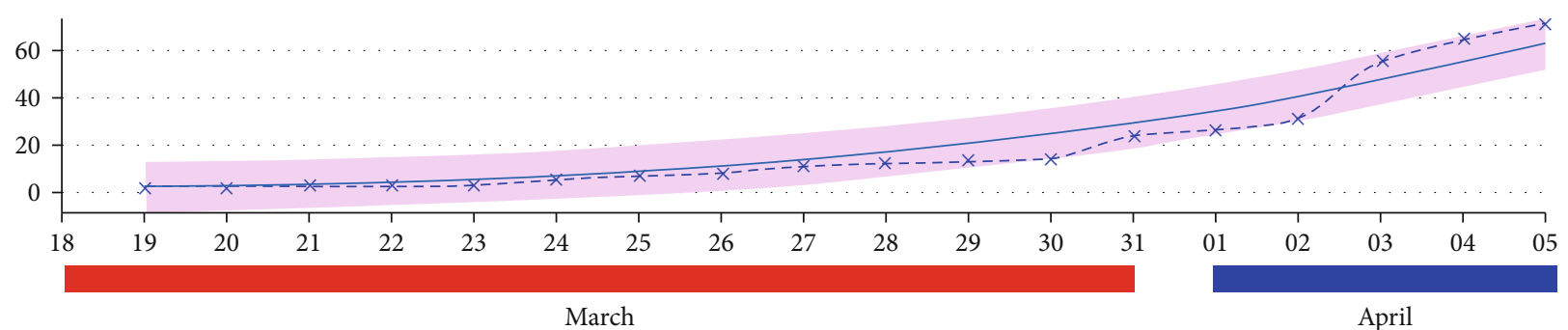

$-*$ - R-data

- Estimated R

Error

(b)

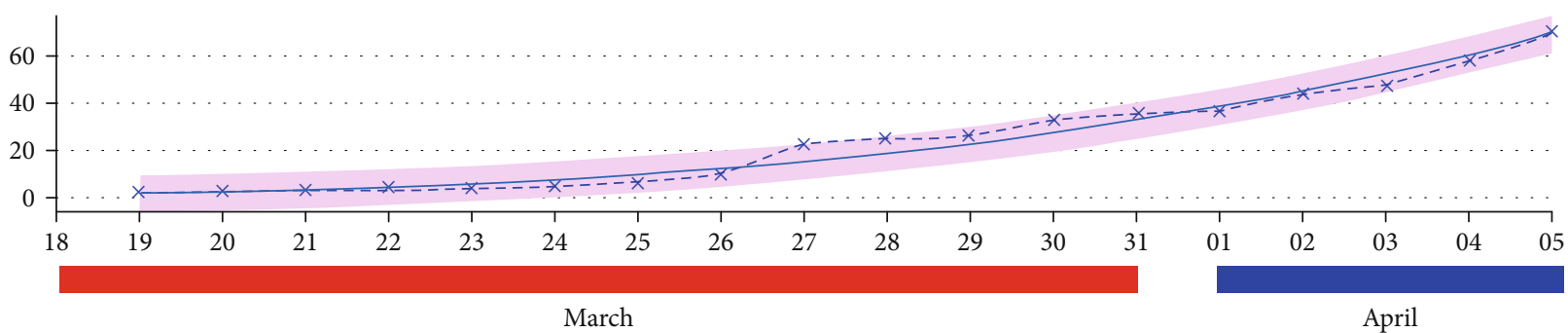

$-*-$ D-data
Estimated D
Error

(c)

FIgURE 1: Estimation of the parameters of the model.

to the actual data. The purpose of this section is to predict what will happen in the coming months and to verify the validity of the proposed model by matching the outputs of the model with the actual data, in order to estimate the high-risk population in Morocco during the period used to estimate the parameters, which will be used to evaluate the control strategy that we will propose later.

\section{An Optimal Control Approach}

In this part of the paper, we propose an optimal strategy of control that helps the authorities in developing countries to bring the situation under control with minimal costs. Authorities provide all necessary assistance to residents to stay at home during the quarantine period, by spending financial values on poor families and by dispensing certain useless occupations during this period. Consequently, people who break quarantine can be considered as violating the laws, as they will take the maximum penalties, which reach the prison.

To test this strategy of control and simulate its effect in Morocco, we introduce in the model a control variable that represents the previous severe reactions of the authorities against people who do not respect the national quarantine, denoted by $u_{i}$. Thus, the controlled model is given by

$$
\begin{aligned}
& S_{i+1}=S_{i}-\frac{\beta}{N_{i}} S_{i} I_{i}-\theta \frac{S_{i}}{1+a S_{i}}+\rho R_{i}-u_{i} S_{i}, \\
& P_{i+1}=P_{i}-\frac{c \beta}{N_{i}} P_{i} I_{i}+\theta_{1} \frac{S_{i}}{1+a S_{i}}-u_{i} P_{i} \\
& C_{i+1}=C_{i}+\theta_{2} \frac{S_{i}}{1+a S_{i}}+u_{i} P_{i}+u_{i} S_{i}
\end{aligned}
$$




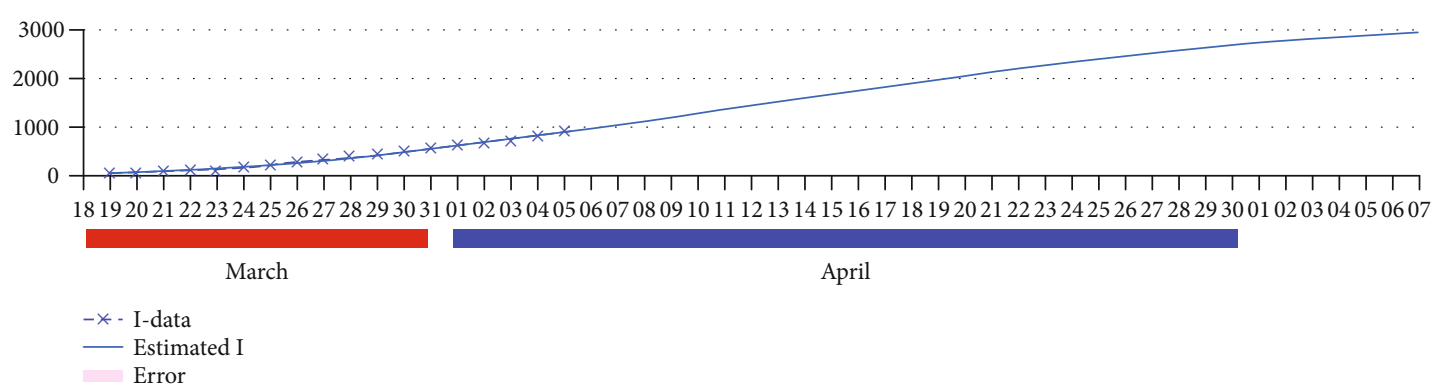

(a)

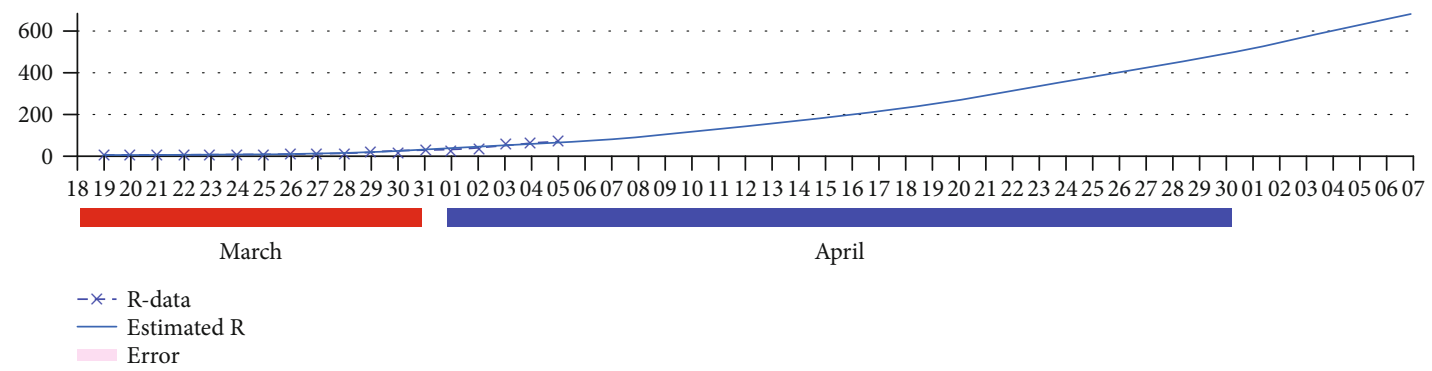

(b)

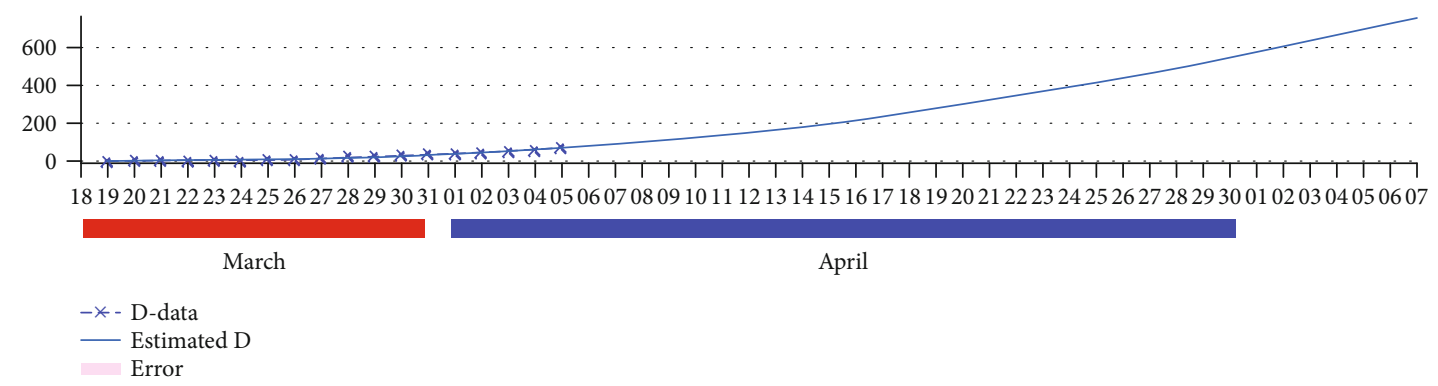

(c)

Figure 2: The model's prediction by April.

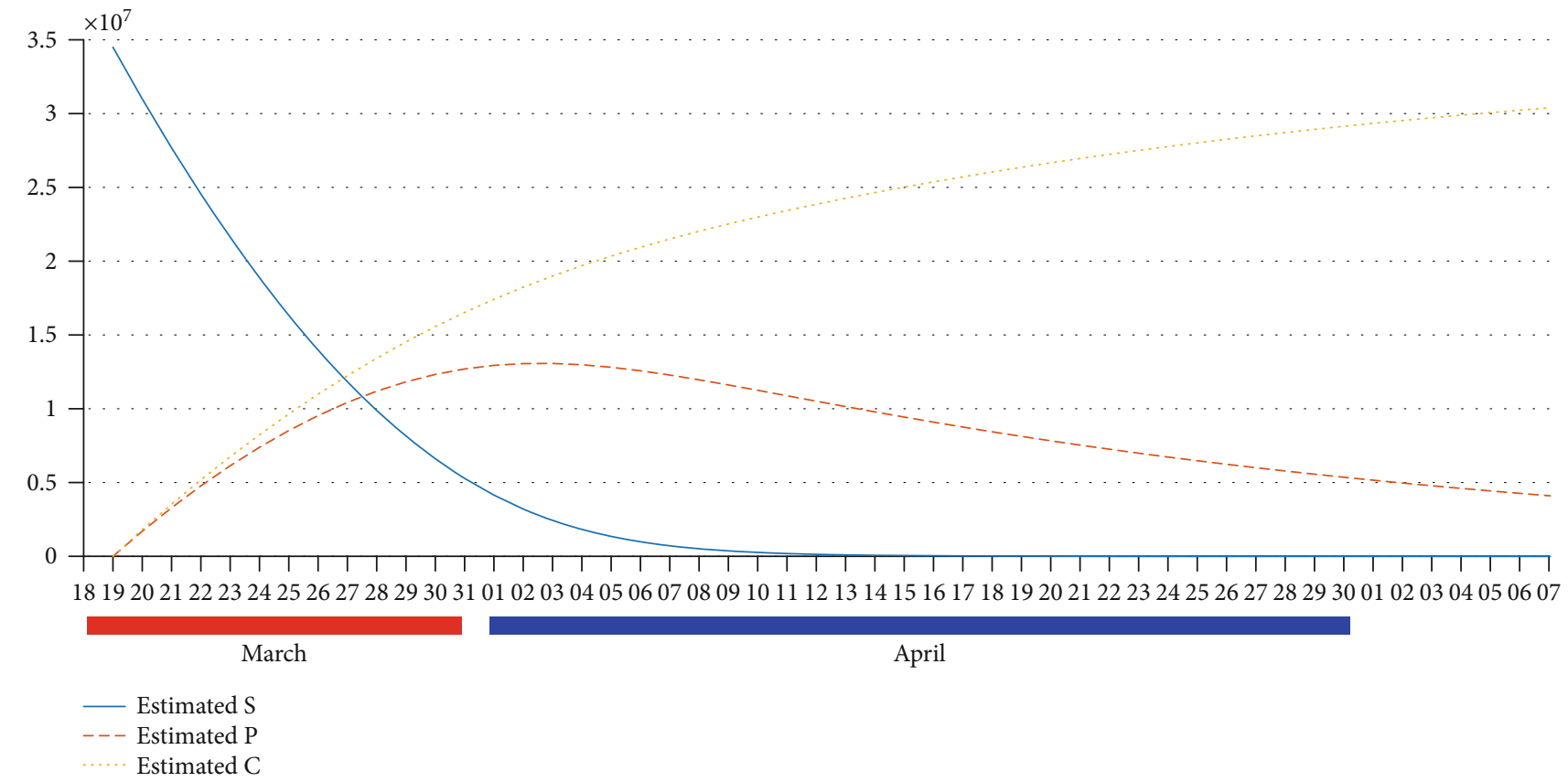

FIGURE 3: Estimation of the partially and totally controlled populations. 


$$
\begin{aligned}
I_{i+1} & =I_{i}+\frac{\beta}{N_{i}}\left(S_{i}+c P_{i}\right) I_{i}-\gamma I_{i}-\alpha I_{i}-\mathscr{T} I_{i}, \\
R_{i+1} & =R_{i}+\mathscr{T} I_{i}+\gamma I_{i}-\rho R_{i}, \\
D_{i+1} & =D_{i}+\alpha I_{i} .
\end{aligned}
$$

with initial conditions $S_{0} \geq 0, P_{0} \geq 0, C_{0} \geq 0, I_{0} \geq 0, R_{0} \geq 0$ , and $D_{0} \geq 0$. Motivated by the desire to reduce the number of infected and the partially controlled people as much as possible while minimizing the cost of applying the control $u$, our objective is to seek an optimal control $u^{*}$ such that:

$$
\mathscr{J}\left(u^{*}\right)=\min _{u \in \mathscr{U}} \mathscr{J}(u)
$$

where $\mathscr{J}$ is the function defined by:

$$
\mathscr{J}(u)=K_{1} I_{T}+K_{2} P_{T}+\sum_{i=0}^{T-1} K_{1} I_{i}+K_{2} P_{i}+\frac{K_{3}}{2} u_{i}^{2}
$$

and where the control space $U$ is defined by the set:

$$
\mathscr{U}=\left\{u \in \mathbb{R}^{T} / u_{\min } \leq u_{i} \leq u_{\max }, \forall i=0, \cdots, T, 0 \leq u_{\min } \leq u_{\max } \leq 1\right\}
$$

$K_{1}, K_{2}$, and $K_{3}$ represent constant severity weights associated with functions $I, P$, and $u$, respectively.

\subsection{Sufficient Conditions}

Theorem 1. There exists an optimal control $u^{*} \in U$ such that

$$
\mathscr{J}\left(u^{*}\right)=\min \{\mathscr{J}(u) / u \in \mathscr{U}\}
$$

subject to the control system (8), (9), (10), (11), (12) and (13) and initial conditions.

4.2. Necessary Conditions. By using a discrete version of Pontryagin's maximum principle [23-27], we derive necessary conditions for our optimal control problem. For this purpose, we define the Hamiltonian as:

$$
\begin{aligned}
\mathscr{H}(i)= & K_{1} I_{i}+K_{2} P_{i}+\frac{K_{3}}{2} u_{i}^{2}+\zeta_{1, i+1} \\
& \cdot\left[S_{i}-\frac{\beta}{N_{i}} S_{i} I_{i}-\theta \frac{S_{i}}{1+a S_{i}}-u_{i} S_{i}+\rho R_{i}\right] \\
& +\zeta_{2, i+1}\left[P_{i}-\frac{c \beta}{N_{i}} P_{i} I_{i}+\theta_{1} \frac{S_{i}}{1+a S_{i}}-u_{i} P_{i}\right] \\
& +\zeta_{3, i+1}\left[C_{i}+\theta_{2} \frac{S_{i}}{1+a S_{i}}+u_{i} P_{i}+u_{i} S_{i}\right] \\
& +\zeta_{4, i+1}\left[I_{i}+\frac{\beta}{N_{i}}\left(S_{i}+c P_{i}\right) I_{i}-\gamma I_{i}-\alpha I_{i}-\mathscr{T} I_{i}\right] \\
& +\zeta_{5, i+1}\left[R_{i}+\mathscr{T} I_{i}+\gamma I_{i}-\rho R_{i}\right]+\zeta_{6, i+1}\left[D_{i}+\alpha I_{i}\right] .
\end{aligned}
$$

Theorem 2. Given an optimal control $u^{*}$ and solutions $S^{*}$, $P^{*}, C^{*}, I^{*}, R^{*}$, and $D^{*}$, there exists $\zeta_{k, i}, i=0 \cdots T-1, k=1,2$ $, \cdots, 6$, the adjoint variables satisfying the following equations:

$$
\begin{aligned}
& \Delta \zeta_{1, i}=\zeta_{4, i+1}\left(\frac{I_{i} S_{i} \beta}{N_{i}^{2}}-\frac{I_{i} \beta}{N_{i}}+\frac{I_{i} P_{i} \beta c}{N_{i}^{2}}\right) \\
& -\zeta_{2, i+1}\left(\frac{\theta_{1}}{S_{i} a+1}-\frac{S_{i} a \theta_{1}}{\left(S_{i} a+1\right)^{2}}+\frac{I_{i} P_{i} \beta c}{N_{i}^{2}}\right) \\
& +\zeta_{1, i+1}\left(u_{i}+\frac{\theta_{1}+\theta_{2}}{S_{i} a+1}+\frac{I_{i} \beta}{N_{i}}-\frac{S_{i} a\left(\theta_{1}+\theta_{2}\right)}{\left(S_{i} a+1\right)^{2}}-\frac{I_{i} S_{i} \beta}{N_{i}^{2}}-1\right) \\
& -\zeta_{3, i+1}\left(u_{i}+\frac{\theta_{2}}{S_{i} a+1}-\frac{S_{i} a \theta_{2}}{\left(S_{i} a+1\right)^{2}}\right) \text {, } \\
& \Delta \zeta_{2, i}=\zeta_{2, i+1}\left(u_{i}+\frac{I_{i} \beta c}{N_{i}}-\frac{I_{i} P_{i} \beta c}{N_{i}^{2}}-1\right) \\
& -\zeta_{3, i+1} u_{i}-K_{2}+\zeta_{4, i+1}\left(\frac{I_{i} S_{i} \beta}{N_{i}^{2}}-\frac{I_{i} \beta c}{N_{i}}+\frac{I_{i} P_{i} \beta c}{N_{i}^{2}}\right) \\
& -\frac{I_{i} S_{i} \beta \zeta_{1, i+1}}{N_{i}^{2}} \\
& \Delta \zeta_{3, i}=\zeta_{4, i+1}\left(\frac{I_{i} S_{i} \beta}{N_{i}^{2}}+\frac{I_{i} P_{i} \beta c}{N_{i}^{2}}\right)-\zeta_{3, i+1}-\frac{I_{i} S_{i} \beta \zeta_{1, i+1}}{N_{i}^{2}} \\
& -\frac{I_{i} P_{i} \beta c \zeta_{2, i+1}}{N_{i}^{2}} \\
& \Delta \zeta_{4, i}=\zeta_{1, i+1}\left(\frac{S_{i} \beta}{N_{i}}-\frac{I_{i} S_{i} \beta}{N_{i}^{2}}\right)-K_{1}-\alpha \zeta_{6, i+1}+\zeta_{2, i+1} \\
& \cdot\left(\frac{P_{i} \beta c}{N_{i}}-\frac{I_{i} P_{i} \beta c}{N_{i}^{2}}\right)-\zeta_{5, i+1}(T+\gamma)+\zeta_{4, i+1} \\
& \text {. }\left(T+\alpha+\gamma-\frac{S_{i} \beta}{N_{i}}+\frac{I_{i} S_{i} \beta}{N_{i}^{2}}-\frac{P_{i} \beta c}{N_{i}}+\frac{I_{i} P_{i} \beta c}{N_{i}^{2}}-1\right) \text {, } \\
& \Delta \zeta_{5, i}=\zeta_{5, i+1}(\rho-1)-\zeta_{1, i+1}\left(\rho+\frac{I_{i} S_{i} \beta}{N_{i}^{2}}\right)+\zeta_{4, i+1} \\
& \cdot\left(\frac{I_{i} S_{i} \beta}{N_{i}^{2}}+\frac{I_{i} P_{i} \beta c}{N_{i}^{2}}\right)-\frac{I_{i} P_{i} \beta c \zeta_{2, i+1}}{N_{i}^{2}},
\end{aligned}
$$

$\Delta \zeta_{6, i}=-\zeta_{6, i+1}$

where $\zeta_{1, T}=\zeta_{3, T}=\zeta_{5, T}=\zeta_{6, T}=0, \zeta_{2, T}=K_{2}, \zeta_{4, T}=K_{1}$ are the transversality conditions. In addition

$$
\begin{aligned}
u_{i}^{*} & =\min \left\{\max \left\{u_{\min }, \frac{P_{i}\left(\zeta_{2, i+1}-\zeta_{3, i+1}\right)+S_{i}\left(\zeta_{1, i+1}-\zeta_{3, i+1}\right)}{K_{3}}\right\}, u_{\max }\right\}, i \\
& =0, \cdots, T-1 .
\end{aligned}
$$

\section{Numerical Simulation and Discussion}

Here, we present numerical simulations associated with the abovementioned optimal control problem. We write a code in MATLAB $^{T M}$ and simulated our results using data from Table 1 . The optimality systems are solved based on an iterative discrete scheme that converges following an appropriate test similar to the one related to the Forward-Backward Sweep Method (FBSM). The state system with an initial guess 


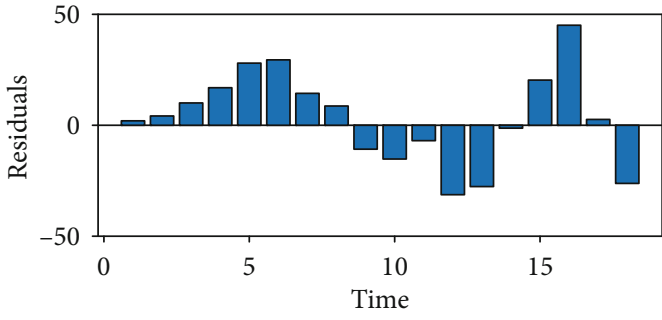

(a)

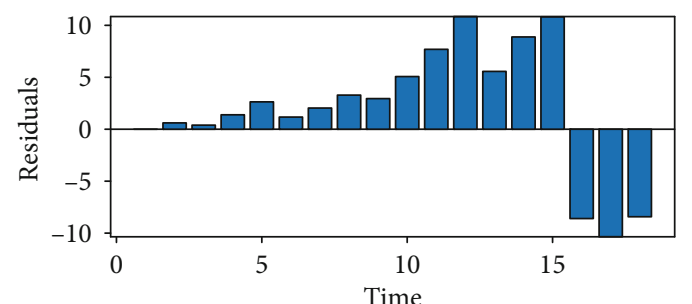

(b)

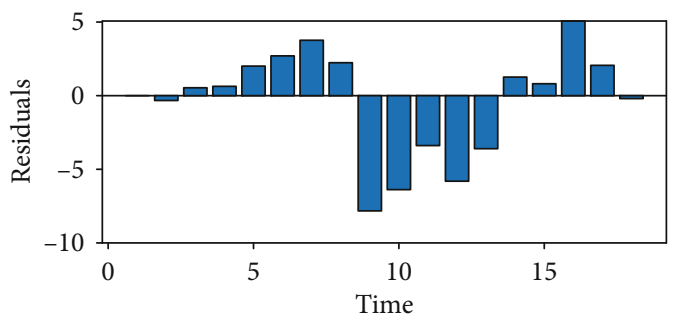

(c)

FIgURE 4: Residuals of the estimation of the parameters.

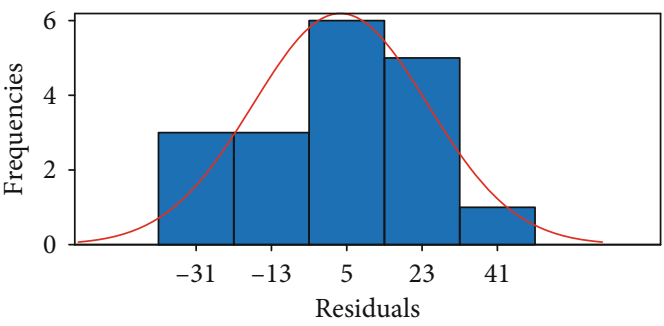

(a)

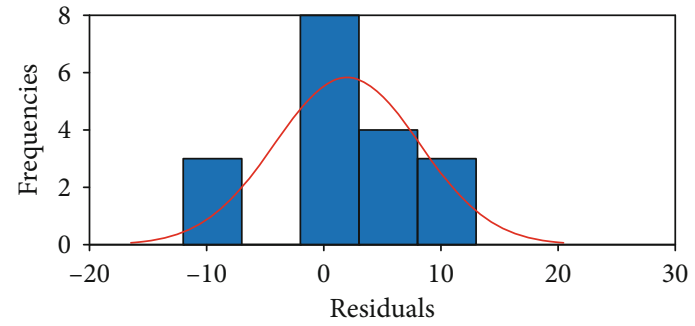

(b)

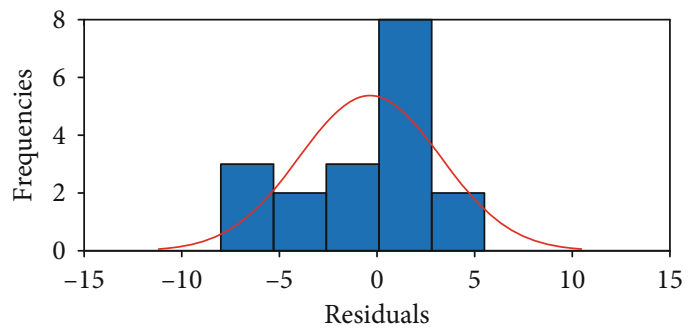

(c)

FIgURE 5: Normality test.

is solved forward in time, and then, the adjoint system is solved backward in time because of the transversality conditions. Afterwards, we update the optimal control values using the values of state and co-state variables obtained at the previous steps. Finally, we execute the previous steps until a tolerance criterion is reached.

Our model correctly corresponds to the actual Moroccan data (see Figure 1) and predicts that the number of COVID19 cases will continue to increase if the Moroccan authorities do not take other measures, see Figure 2.

While in Figure 6, we can see that the number of infections reaches the peak by about 130 infected individuals on
March 26 and then starts to decrease. The number of deaths does not exceed 60 individuals (see the subfigure (b)) by the first week of May. By following this strategy of control, the number of partially controlled individuals does not exceed 4 million individuals to start decreasing after about 5 days from the beginning of the closure on March 20. This number is almost null by the first week of April (see the subfigure (a)), compared to the case when the control $u_{i}$ takes a fixed value from the beginning of the quarantine, whereas the number of people under partial control reaches about 15 million and maintains values of not less than 5 million until the end of the simulation by May 07 . 


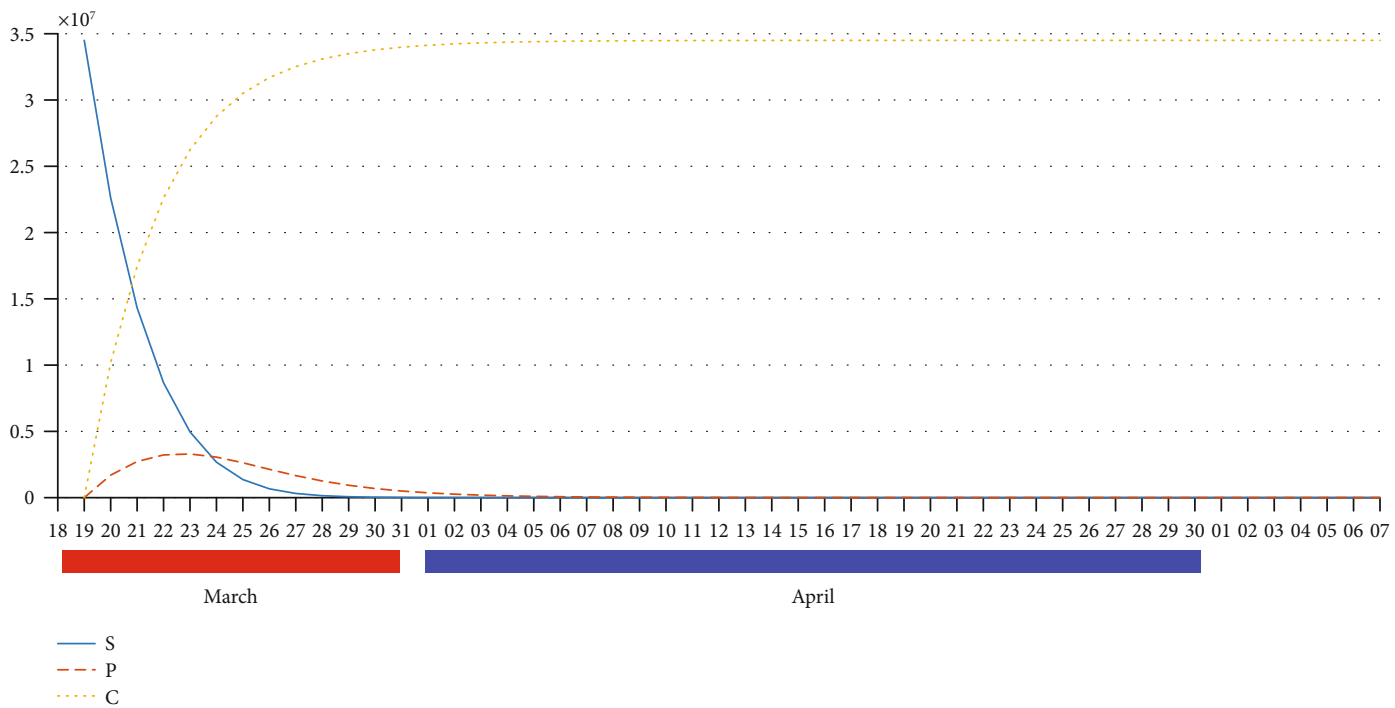

(a)

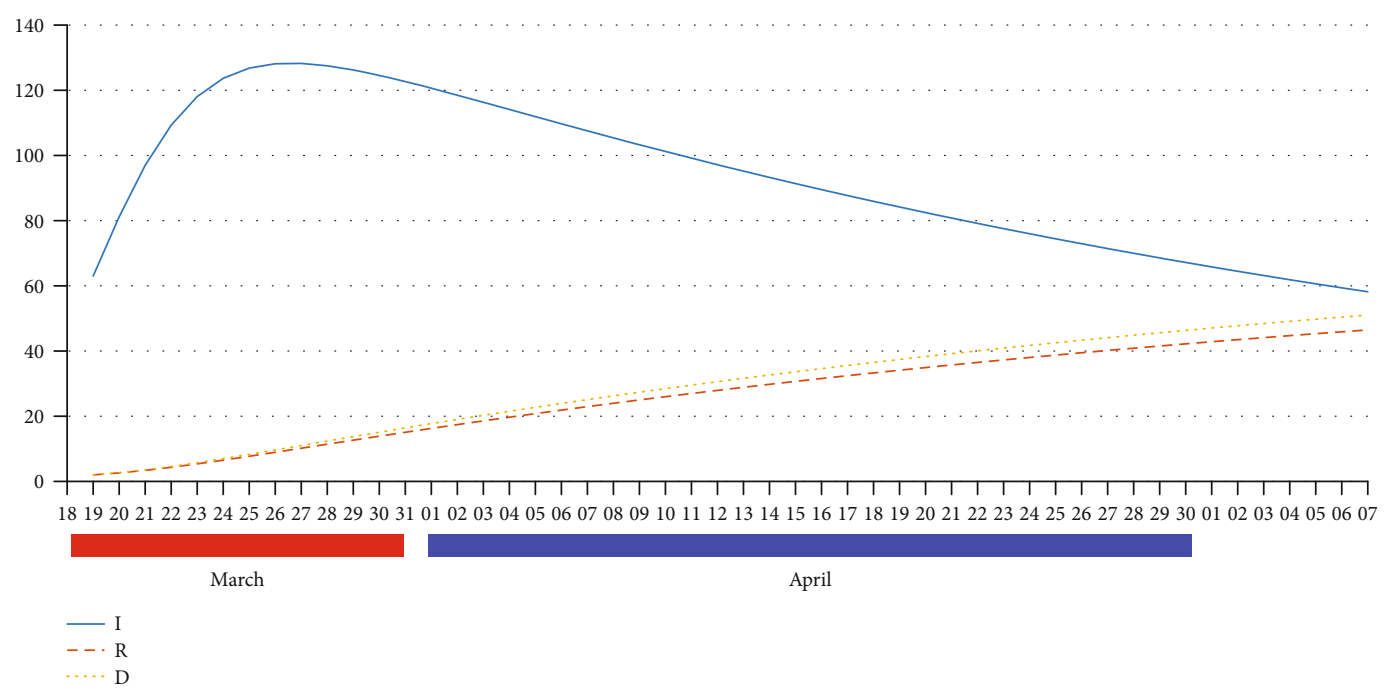

(b)

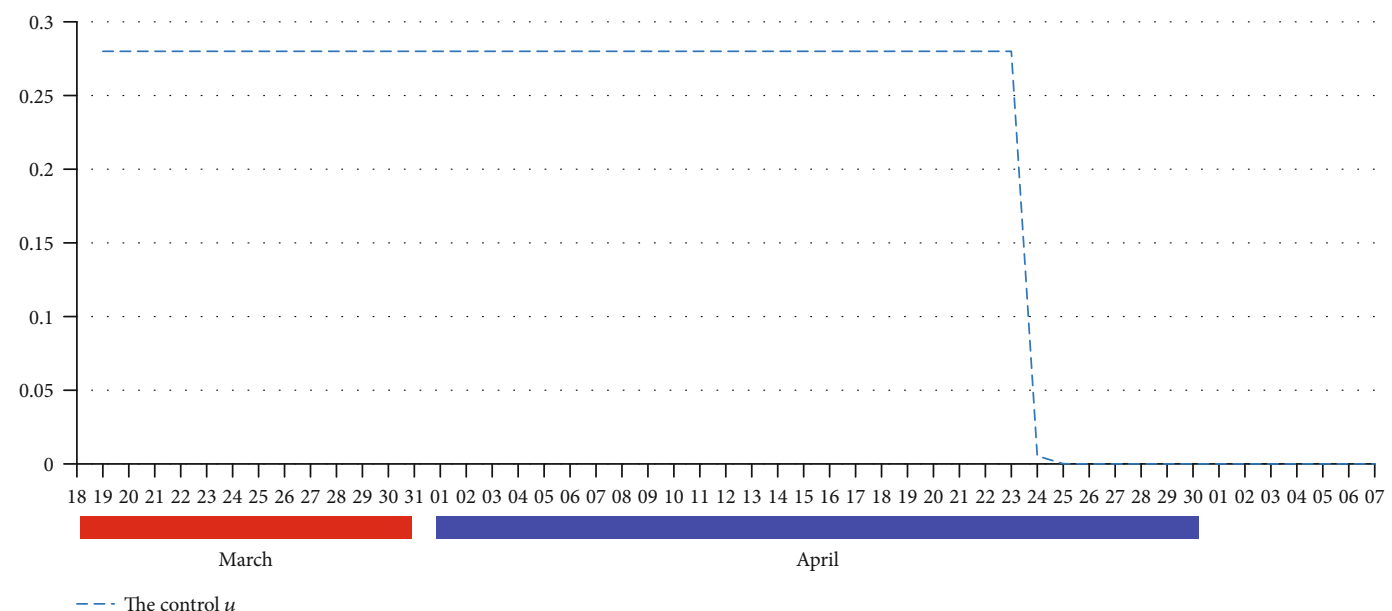

(c)

FIGURE 6: States of the controlled model (8), (9), (10), (11), (12) and (13) where the objective function given in (14) is equal to 39.2. (a) The three functions of susceptible $S$, partially controlled $P$, and totally controlled $C$ population. (b) The three functions of infected $I$, removed $R$, and dead $D$ population. (c) The control function $u$. 
TABLE 2: PRCCs for $J\left(u^{*}\right)$ and the model's parameters.

\begin{tabular}{lcc}
\hline Parameter & PRCC & $p$ value \\
\hline$\beta$ & 0.4832 & $4.2704 \times 10^{-13}$ \\
$c$ & 0.4176 & $7.6509 \times 10^{-10}$ \\
$\theta_{1}$ & 0.0502 & 0.4806 \\
$\theta_{2}$ & -0.1998 & 0.0046 \\
$\alpha$ & -0.0283 & 0.6910 \\
$T$ & -0.0856 & 0.2280 \\
$\gamma$ & -0.0521 & 0.4640 \\
$\rho$ & 0.0165 & 0.8164 \\
$a$ & 0.0174 & 0.8068 \\
\hline
\end{tabular}

It can be seen also from this subfigure that by using the control strategy we are proposing here, the number of totally controlled individuals rises towards 34 million by the end of March, which means that the population at risk is reduced as much as possible before the month of April.

While it can be seen, from the subfigure (c) of Figure 6, that the $u_{i}$ control function takes a constant value of about 0.28 until the last week of April to continue with null values. It can also be observed that optimal control is required approximately three weeks after the disappearance of the partially controlled population, to ensure a continuous decline of active cases.

The lack of effective treatment and vaccines against COVID-19 and the limited medical resources are all factors that make it more difficult to control the transmission of this pandemic. Therefore, there is a need to consider other means of intervention such as nonmedical interventions, especially in developing countries. Travel restrictions have been implemented in all regions of Morocco, effectively reducing the movement of millions; quarantine and state health emergencies force people to stay indoors during the COVID-19 epidemic to reduce possible contact, which already contributes to the prevention and control of the disease in the country, compared to other affected countries. With all these efforts and control strategies, the new cases of infection still registered in Morocco, which gives us the opportunity to contribute to the containment of this dangerous pandemic by proposing the optimal strategy of control which appears, from numerical simulation, to be more efficient by bringing forward the peak and reducing the size of the infection peak in the country.

Although this is the first work using a mathematical model to estimate the population that respects or does not respect the national closure, several limits must be observed. Since we can estimate the population at high risk in countries subject to emergency and quarantine situations in general, and in Morocco in particular, there are potential limitations regarding the long-term risk of COVID-19. As the risk of developing COVID-19 mainly depends on age, gender, and other diseases (obesity, diabetes, heart disease, lung disease, and kidney disease) [28], therefore, other results may be when assessing the development of COVID-19 in these individuals or when considering other models that include these factors. However, we assessed the prevalence of COVID-19 in this country independently of these factors because among the objectives of this contribution is the estimation of a population at high risk regardless of their state of health. In addition, data collection errors reduce the performance of the model or the possibility of overestimating future expectations. Another limitation is that the authorities often change their control strategy by adding additional controls such as the mask requirement, more movement restrictions, and massive tests-all of which can lead to large errors in predictions of the model. However, our optimal control strategy is even more effective even if there are different initial values for the population. Where it is possible through numerical simulation that the control strategy, we propose can effectively reduce the number of infections and deaths.

\section{Global Sensitivity Analysis}

In order to search for the parameters that are key factors to the spread of COVID-19, we perform in this section a global sensitivity analysis. We assess the impact of variations in the model's parameters on the outputs $J\left(u^{*}\right)$ using the Partial Rank Correlation Coefficient (PRCC) [29]. These coefficients are similar to the common Pearson Correlation Coefficient, but also deal with nonlinearities, as long as the output is monotonic in the parameters.

The Latin Hypercube Sampling (LHS) was simulated with 400 trials, and parameters from model (1) were considered following normal distributions, with baseline values given by Table 1 . Ranges were chosen corresponding to $15 \%$ of maximum variation from their baselines. The normal distributions are within these ranges. We considered a significance level of 0.01 , meaning that PRCCs with $p$ values greater than 0.01 are not significant. PRCC results and $p$ values considering the cost $J\left(u^{*}\right)$ as output are shown in Table 2.

We found that the infection rate $\beta$ and the reduced chances of a partially controlled individual to be infected $c$ are positively correlated with $J\left(u^{*}\right)$, while the recruitment rate of susceptibles to the totally controlled class parameter $\theta_{1}$ negatively correlated to $J\left(u^{*}\right)$.

\section{Conclusion}

In this paper, a new mathematical model describing the evolution of the COVID-19 in countries subject to the state of a health emergency is considered. Based on the classical compartmental models, two new compartments are added to the classical SIRS model, representing the people who have benefited from the awareness programs and they could not respect the national quarantine for their personal reasons, we called them the partially controlled people. The second are people who know about the pandemic and respect the lockdown by staying at home, which we called the totally controlled 
people. The impact of these populations on the severity of the COVID-19 infection is investigated. Using real COVID-19 propagation data in Morocco collected and published by the Johns Hopkins University, we estimated the parameters of the model, and then, we estimated the totally and partially controlled populations. We found that the number of totally controlled and partially controlled people rose slowly from the beginning of the national lockdown. We found that the population at risk in Morocco is more than 10 million individuals in the first month of the closure, and then the need for an efficient control strategy to save more lives. A new strategy of optimal control is investigated, which aimed to reduce the number of partially controlled individuals and then reaching the peak of the infection at a very small number. Numerical simulations are provided throughout the paper to illustrate the efficiency of our study and the strategy of control we propose.

\section{Appendix}

\section{Proofs of Theorems}

Proof of Theorem 3. Since the parameters of the system are bounded and there are a finite number of time steps, that is $S, P, C, I, R$, and $D$ are uniformly bounded for all $u$ in the control set $\mathrm{U}$, thus $\mathrm{J}(u)$ is also bounded for all $u \in \mathrm{U}$, which implies that $\inf _{u \in U} J(u)$ is finite, and there exists a sequence $u^{n} \in U$ such that

$$
\lim _{n \rightarrow+\infty} \mathscr{J}\left(u^{n}\right)=\inf _{u \in \mathscr{U}} \mathscr{J}(u)
$$

and corresponding sequences of states, $S^{n}, P^{n}, C^{n}, I^{n}, R^{n}$, and $D^{n}$.

Since there is a finite number of uniformly bounded sequences, there exists $u^{*} \in U$ and $S^{*}, P^{*}, C^{*}, I^{*}, R^{*}$, and $D^{*}$ such that, on a sequence,

$$
\begin{gathered}
u^{n} \longrightarrow u^{*}, \\
I^{n} \longrightarrow I^{*}, \\
S^{n} \longrightarrow S^{*}, \\
R^{n} \longrightarrow R^{*}, \\
F^{n} \longrightarrow F^{*} .
\end{gathered}
$$

Finally, due to the finite dimensional structure of the system (8), (9), (10), (11), (12) and (13) and the objective function $J(u), u^{*}$ is an optimal control with corresponding states $S^{*}, P^{*}, C^{*}, I^{*}, R^{*}$, and $D^{*}$, which completes the proof.

Proof of Theorem 4. Using the discrete version of Pontryagin's maximum principle $[23,24]$, we obtain the following adjoint equations:

$$
\begin{aligned}
\Delta \zeta_{1, i}= & -\frac{\partial \mathscr{H}}{\partial S_{i}}=\zeta_{4, i+1}\left(\frac{I_{i} S_{i} \beta}{N_{i}^{2}}-\frac{I_{i} \beta}{N_{i}}+\frac{I_{i} P_{i} \beta c}{N_{i}^{2}}\right) \\
& -\zeta_{2, i+1}\left(\frac{\theta_{1}}{S_{i} a+1}-\frac{S_{i} a \theta_{1}}{\left(S_{i} a+1\right)^{2}}+\frac{I_{i} P_{i} \beta c}{N_{i}^{2}}\right) \\
& +\zeta_{1, i+1}\left(u_{i}+\frac{\theta_{1}+\theta_{2}}{S_{i} a+1}+\frac{I_{i} \beta}{N_{i}}-\frac{S_{i} a\left(\theta_{1}+\theta_{2}\right)}{\left(S_{i} a+1\right)^{2}}-\frac{I_{i} S_{i} \beta}{N_{i}^{2}}-1\right) \\
& -\zeta_{3, i+1}\left(u_{i}+\frac{\theta_{2}}{S_{i} a+1}-\frac{S_{i} a \theta_{2}}{\left.\left(S_{i} a+1\right)^{2}\right)}\right) \\
\Delta \zeta_{2, i}= & -\frac{\partial \mathscr{H}}{\partial P_{i}}=\zeta_{2, i+1}\left(u_{i}+\frac{I_{i} \beta c}{N_{i}}-\frac{I_{i} P_{i} \beta c}{N_{i}^{2}}-1\right) \\
& -\zeta_{3, i+1} u_{i}-K_{2}+\zeta_{4, i+1}\left(\frac{I_{i} S_{i} \beta}{N_{i}^{2}}-\frac{I_{i} \beta c}{N_{i}}+\frac{I_{i} P_{i} \beta c}{N_{i}^{2}}\right) \\
& -\frac{I_{i} S_{i} \beta \zeta_{1, i+1}}{N_{i}^{2}},
\end{aligned}
$$$$
\begin{aligned}
\Delta \zeta_{3, i}= & -\frac{\partial \mathscr{H}}{\partial C_{i}}=\zeta_{4, i+1}\left(\frac{I_{i} S_{i} \beta}{N_{i}^{2}}+\frac{I_{i} P_{i} \beta c}{N_{i}^{2}}\right)-\zeta_{3, i+1}-\frac{I_{i} S_{i} \beta \zeta_{1, i+1}}{N_{i}^{2}} \\
& -\frac{I_{i} P_{i} \beta c \zeta_{2, i+1}}{N_{i}^{2}}, \\
\Delta \zeta_{4, i}= & -\frac{\partial \mathscr{H}}{\partial I_{i}}=\zeta_{1, i+1}\left(\frac{S_{i} \beta}{N_{i}}-\frac{I_{i} S_{i} \beta}{N_{i}^{2}}\right)-K_{1}-\alpha \zeta_{6, i+1} \\
& +\zeta_{2, i+1}\left(\frac{P_{i} \beta c}{N_{i}}-\frac{I_{i} P_{i} \beta c}{N_{i}^{2}}\right)-\zeta_{5, i+1}(T+\gamma)+\zeta_{4, i+1} \\
& \cdot\left(T+\alpha+\gamma-\frac{S_{i} \beta}{N_{i}}+\frac{I_{i} S_{i} \beta}{N_{i}^{2}}-\frac{P_{i} \beta c}{N_{i}}+\frac{I_{i} P_{i} \beta c}{N_{i}^{2}}-1\right),
\end{aligned}
$$$$
\Delta \zeta_{5, i}=-\frac{\partial \mathscr{H}}{\partial R_{i}}=\zeta_{5, i+1}(\rho-1)-\zeta_{1, i+1}\left(\rho+\frac{I_{i} S_{i} \beta}{N_{i}^{2}}\right)+\zeta_{4, i+1}
$$$$
\cdot\left(\frac{I_{i} S_{i} \beta}{N_{i}^{2}}+\frac{I_{i} P_{i} \beta c}{N_{i}^{2}}\right)-\frac{I_{i} P_{i} \beta c \zeta_{2, i+1}}{N_{i}^{2}},
$$

$\Delta \zeta_{6, i}=-\frac{\partial \mathscr{H}}{\partial D_{i}}=-\zeta_{6, i+1}$,

with $\quad \zeta_{1, T}=\zeta_{3, T}=\zeta_{5, T}=\zeta_{6, T}=0, \zeta_{2, T}=K_{2}, \zeta_{4, T}=K_{1} . \quad$ To obtain the optimality conditions, we take the variation with respect to controls $\left(u_{i}\right.$ and $\left.v_{i}\right)$ and set it equal to zero

$$
\frac{\partial \mathscr{H}}{\partial u_{i}}=P_{i} \zeta_{2, i+1}+S_{i} \zeta_{1, i+1}-K_{3} u_{i}-\zeta_{3, i+1}\left(P_{i}+S_{i}\right)=0 .
$$

Then, we obtain the optimal control

$$
u_{i}=\frac{P_{i}\left(\zeta_{2, i+1}-\zeta_{3, i+1}\right)+S_{i}\left(\zeta_{1, i+1}-\zeta_{3, i+1}\right)}{K_{3}} .
$$

By the bounds in $U$ of the control in the definitions (16), it is easy to obtain $u^{*}{ }_{i}$ in the following form 


$$
\begin{aligned}
u_{i}^{*} & =\min \left\{\max \left\{u_{\min }, \frac{P_{i}\left(\zeta_{2, i+1}-\zeta_{3, i+1}\right)+S_{i}\left(\zeta_{1, i+1}-\zeta_{3, i+1}\right)}{K_{3}}\right\}, u_{\max }\right\}, i \\
& =0, \cdots, T-1
\end{aligned}
$$

\section{Data Availability}

The data of [6] is used to support this study.

\section{Conflicts of Interest}

The authors declare that they have no conflicts of interest.

\section{Acknowledgments}

The authors would like to thank all the members of the Editorial Board who were responsible for this paper, and the anonymous referees for their valuable comments and suggestions to improve the content of this paper.

\section{References}

[1] World Health Organization, "Coronavirus," https://www.who .int/fr/health-topics/coronavirus/coronavirus.

[2] World Health Organization, "Q and a on coronaviruses," https://www.who.int/fr/news-room/q-a-detail/qacoronaviruses.

[3] O. Zakary, M. Rachik, and I. Elmouki, "A new epidemic modeling approach: multi-regions discrete-time model with travel-blocking vicinity optimal control strategy," Infectious Disease Modelling, vol. 2, no. 3, pp. 304-322, 2017.

[4] O. Zakary, M. Rachik, and I. Elmouki, "A multi-regional epidemic model for controlling the spread of ebola: awareness, treatment, and travel-blocking optimal control approaches," Mathematical Methods in the Applied Sciences, vol. 40, no. 4, pp. 1265-1279, 2017.

[5] O. Zakary, A. Larrache, M. Rachik, and I. Elmouki, "Effect of awareness programs and travel-blocking operations in the control of hiv/aids outbreaks: a multi-domains sir model," Advances in Difference Equations, vol. 2016, no. 1, 2016.

[6] Johns Hopkins University Center for Systems Science and Engineering (JHU CCSE), "Realtime data of the new coronavirus," 2020.

[7] N. Zhu, D. Zhang, W. Wang et al., "A novel coronavirus from patients with pneumonia in China, 2019," The New England Journal of Medicine, vol. 382, no. 8, pp. 727-733, 2020.

[8] J. Nkengasong, “Author Correction: China's response to a novel coronavirus stands in stark contrast to the 2002 SARS outbreak response," Nature Medicine, vol. 26, no. 3, p. 441, 2020.

[9] V. C. C. Cheng, S.-C. Wong, K. K. W. To, P. L. Ho, and K.Y. Yuen, "Preparedness and proactive infection control measures against the emerging novel coronavirus in china," Journal of Hospital Infection, vol. 104, no. 3, pp. 254-255, 2020.

[10] V. C. C. Cheng, J. W. M. Tai, L. M. W. Wong et al., "Prevention of nosocomial transmission of swine-origin pandemic influenza virus a/h1n1 by infection control bundle," Journal of Hospital Infection, vol. 74, no. 3, pp. 271-277, 2010.

[11] V. C. C. Cheng, K. K. W. To, H. Tse, I. F. N. Hung, and K. Y. Yuen, "Two years after pandemic influenza a/2009/h1n1: what have we learned?," Clinical Microbiology Reviews, vol. 25, no. 2, pp. 223-263, 2012.

[12] V. C. C. Cheng, J. W. M. Tai, W. M. Lee et al., "Infection control preparedness for human infection with influenza a h7n9 in hong kong," Infection Control \& Hospital Epidemiology, vol. 36, no. 1, pp. 87-92, 2015.

[13] "Higher Education Kingdom of Morocco Ministry of National Education, Vocational Training and Scientific Research. Press release - march 16, 2020," 2020, https://www.men.gov.ma/Ar/ Pages/Publication.aspx?IDPublication $=5938$.

[14] "Higher Education Kingdom of Morocco Ministry of National Education, Vocational Training and Scientific Research. Press release - march 16, 2020 newsletter no. 3," 2020, https://www .men.gov.ma/Ar/Pages/Publication.aspx?IDPublication=5941.

[15] B. Khajji, D. Kada, O. Balatif, and M. Rachik, "A multi-region discrete time mathematical modeling of the dynamics of Covid-19 virus propagation using optimal control," Journal of Applied Mathematics and Computing, vol. 64, no. 1-2, pp. 255-281, 2020.

[16] M. Kamrujjaman, U. Ghosh, and M. S. Islam, "Pandemic and the dynamics of seir model: Case covid-19," 2020.

[17] D. Sinha and N. Klahn, "Mathematical modeling study of the 2020 covid-19 outbreak in the United States," SSRN Electronic Journal, 2020.

[18] A. S. Shaikh, I. N. Shaikh, and K. S. Nisar, "A mathematical model of covid-19 using fractional derivative: outbreak in India with dynamics of transmission and control," Advances in Difference Equations, vol. 2020, no. 1, 2020.

[19] A. Kouidere, B. Khajji, A. El Bhih, O. Balatif, and M. Rachik, "A mathematical modeling with optimal control strategy of transmission of covid-19 pandemic virus," Communications in Mathematical Biology and Neuroscience, vol. 2020, 2020.

[20] the Center for Systems Science and Engineering (CSSE) at Johns Hopkins University (JHU), "Novel coronavirus (covid19) cases," 2020, https://github.com/CSSEGISandData/ COVID-19.

[21] M. L. Michelsen, P. G. Thomsen, and M. R. Kristensen, Parameter Estimation in Nonlinear Dynamical Systems, Department of Chemical Engineering Technical University of Denmark. Master's thesis, 2004, http://www .rodekristensen.dk/MasterThesisMRK.pdf.

[22] A. R. Conn, N. I. M. Gould, and P. L. Toint, Trust Region Methods, SIAM, 2000.

[23] L. S. Pontryagin, Mathematical Theory of Optimal Processes, Routledge, 2018.

[24] S. P. Sethi and G. L. Thompson, Optimal Control Theory: Applications to Management Science and Economics, Springer, 2000.

[25] O. Zakary, M. Rachik, and I. Elmouki, "On the analysis of a multi-regions discrete sir epidemic model: an optimal control approach," International Journal of Dynamics and Control, vol. 5, no. 3, pp. 917-930, 2017.

[26] O. Zakary, M. Rachik, and I. Elmouki, "A new analysis of infection dynamics: multi-regions discrete epidemic model with an extended optimal control approach," International Journal of Dynamics and Control 5.4, pp. 1010-1019, 2017.

[27] H. Boutayeb, S. Bidah, O. Zakary, and M. Rachik, "A new simple epidemic discrete-time model describing the dissemination of information with optimal control strategy," Discrete Dynamics in Nature and Society, vol. 2020, Article ID 7465761, 11 pages, 2020. 
[28] L. D'Antiga, "Coronaviruses and immunosuppressed patients: the facts during the third epidemic," Liver Transplantation, vol. 26, no. 6, pp. 832-834, 2020.

[29] S. Bidah, O. Zakary, and M. Rachik, "Stability and global sensitivity analysis for an agree-disagree model: partial rank correlation coefficient and latin hypercube sampling methods," International Journal of Differential Equations, vol. 2020, Article ID 5051248, 14 pages, 2020. 